\title{
Correlations of Peaks of Gaussian Random Fields ${ }^{\star}$
}

\author{
James M. Cline, H. David Politzer, Soo-Jong Rey ${ }^{\star \star}$, and Mark B. Wise ${ }^{\star \star}$ \\ California Institute of Technology, Pasadena, CA 91125, USA
}

\begin{abstract}
The high peaks of a Gaussian random field are studied. Asymptotic expansions, appropriate for high peak thresholds and large spatial separations, are developed for the $N$-point correlation functions of the number density of high peaks, in terms of the two-point correlation of the underlying Gaussian field. Similar expressions are derived for the correlations of points, not necessarily the positions of peaks, where the field exceeds a high threshold.
\end{abstract}

\section{Introduction}

The mathematical modeling of many physical processes often proceeds via a statistical approach. The behavior of the random field of interest, $\varepsilon(\vec{x})$, is described by a probability distribution $P[\varepsilon]$, or equivalently by the moments of the probability distribution (we assume here that all the moments exist),

$$
\left\langle e\left(\vec{x}_{1}\right) \ldots \varepsilon\left(\vec{x}_{n}\right)\right\rangle=\int[d \varepsilon] \varepsilon\left(\vec{x}_{1}\right) \ldots \varepsilon\left(\vec{x}_{n}\right) P[\varepsilon] .
$$

In Eq. (1) the $\vec{x}_{i}$ are points in a $D$-dimensional Euclidean space $\mathbb{R}^{D}$, and the integration is over the value of $\varepsilon$ at each point in $\mathbb{R}^{D}$. Some applications have a random variable which depends only on time, in which case $D=1$. This occurs, for example, in the theory of noise in electrical networks [1]. Other applications may deal with a field $\varepsilon$ which takes values that depend on the location in "physical" space, in which case $D=3$. One such example is the theory of the large scale structure of the universe, where $\varepsilon$ is the mass density fluctuation field [2,3].

The most common probability distribution encountered in practice is a Gaussian distribution, which has

$$
P[\varepsilon]=\frac{1}{Z} \exp \left[-\frac{1}{2} \int d^{D} x d^{D} y \varepsilon(\vec{x}) \xi^{-1}(|\vec{x}-\vec{y}|) \varepsilon(\vec{y})\right],
$$

where $Z$ is a constant chosen so that $P[\varepsilon]$ is normalized to unity and $\xi^{-1}(|\vec{x}-\vec{y}|)$ is

\footnotetext{
* Work supported in part by U.S. Department of Energy under contract DEAC03-81-ER40050.

$\star \star$ KFAS Graduate Fellow

$\star \star \star$ Alfred P. Sloan Foundation Fellow and supported in part by U.S. Department of Energy

Outstanding Junior Investigator Program under contract No. DE-FG03-84 ER40172
} 
the inverse of the two-point correlation,

$$
\begin{gathered}
\langle\varepsilon(\vec{x}) \varepsilon(\vec{y})\rangle=\xi(|\vec{x}-\vec{y}|), \\
\int d^{D} \vec{y} \xi(|\vec{x}-\vec{y}|) \xi^{-1}(|\vec{y}-\vec{z}|)=\delta^{D}(\vec{x}-\vec{z}) .
\end{gathered}
$$

We assume homogeneity and isotropy in the $D$-dimensional Euclidean space so that $\xi$ is only a function of $|\vec{x}-\vec{y}|$. For a Gaussian distribution all the connected $N$-point correlations of $\varepsilon$ vanish for $N>2$.

Often one is interested not in typical values of $\varepsilon$ but only in the local maxima of $\varepsilon(\vec{x})$ that are above some high threshold. For instance, in the theory of biased galaxy formation, high peaks of the mass density field ${ }^{1}$ are taken as the sites where bright galaxies or rich clusters of galaxies form. Even if the underlying field $\varepsilon(\vec{x})$ is Gaussian distributed, the peaks of this field that are above a high threshold are described by a number density $n_{m}(\vec{x})$ that is not a Gaussian random variable. In this paper we develop a systematic expansion for the $N$-point correlations of $n_{m}(\vec{x})$ at large thresholds and large distances (assuming that $\xi(|\vec{x}-\vec{y}|) / \xi(0)$ goes to zero at large separations and that $\xi$ and its first four derivatives exist).

Our presentation is organized as follows. In Sect. II, we construct formulae that generate, by differentiation, the asymptotic expansion ${ }^{2}$ in high threshold, $T$, for the correlation of points $\vec{x}_{i}$ such that $\varepsilon\left(\vec{x}_{i}\right) \geqq \sqrt{T \xi(0)}$ (Eqs. (9) and (12)). (Note that $T$ is dimensionless). Examples are given of the explicit results to $O\left(T^{-2}\right)$ (Eq. (13)) and for the leading terms in joint expansions in $T^{-1}$ and $\xi(x) / \xi(0)$ (presumed small at large separations) (Eqs. $(14,15))$. Peaks above threshold require the further constraints that $\vec{\nabla} \varepsilon$ vanish at the positions of the peaks and that $\vec{\nabla} \vec{\nabla} \varepsilon$ have negative eigenvalues there. The former constraint is straightforward to implement while the latter is quite complex. We defer until Sect. IV an explicit estimate of the error made by ignoring the restriction to negative eigenvalues of $\vec{\nabla} \vec{\nabla} \varepsilon$ for large $T$ and simply proceed in Sect. III on the assumption that essentially all relevant extrema above a large $T$ are indeed peaks. Since the formulae become cumbersome, we exhibit explicitly only terms down to relative order $T^{-2}$ and $\xi(x) / \xi(0)$. Equations (55) and (57) are our principal results. In Sect. IV, we give an error estimate for the preceding calculations. We find that our approximation is valid up to corrections of relative order $\exp \left(-T \xi^{2}(0) / \xi^{2}(x)\right) / \exp (-T)$ and $\exp (-T)$, where $x$ is a typical separation. For the density of peaks, we find that peaks far outnumber local minima and saddle points, by a factor of order $\exp (T)$. The issues for correlations are considerably more subtle. Since minima above threshold, rare as they may be, are strongly correlated with nearby maxima, simple results are obtained only for large separations in the high $T$ expansion.

\section{Large Values of a Gaussian Random Field}

The probability $P_{N}\left(\vec{x}_{1}, \ldots, \vec{x}_{N}\right)$ that the Gaussian random field $\varepsilon(\vec{x})$ will simultaneously exceed some threshold $t=[T \xi(0)]^{1 / 2}$ at the points $\vec{x}_{i}, i=1, \ldots, N$ is given by

1 Filtered so as to smooth out very short wavelength fluctuations

2 Throughout this paper the expansions are presumed, though not proven, to be asymptotic 
[4]

$$
P_{N}\left(\vec{x}_{1}, \ldots, \vec{x}_{N}\right)=\int_{t}^{\infty} d k_{1} \ldots \int_{t}^{\infty} d k_{N} \int[d \varepsilon] P[\varepsilon] \prod_{i=1}^{N} \delta\left(\varepsilon\left(\vec{x}_{i}\right)-k_{i}\right) .
$$

With $P[\varepsilon]$ given by Eq. (2) the functional integration gives [4]

$$
P_{N}\left(\vec{x}_{1}, \ldots, \vec{x}_{N}\right)=\frac{(\operatorname{det} \tilde{M})^{-1 / 2}}{(2 \pi)^{N / 2}} \int_{\vec{t}}^{\infty} d^{N} k \exp \left(-\frac{1}{2} \vec{k}^{T} \tilde{M}^{-1} \vec{k}\right)
$$

where $\vec{t}=(t, t, \ldots, t), \vec{k}=\left(k_{1}, \ldots, k_{n}\right)$ and the symmetric $N \times N$ matrix $\tilde{M}$ has components $\tilde{M}_{i j}=\xi\left(\left|\vec{x}_{i}-\vec{x}_{j}\right|\right)$. It is convenient to change integration variables to $\vec{\lambda}=T(\vec{k} / t-\overrightarrow{1})$, where $\overrightarrow{1}=(1, \ldots, 1)$, and to introduce the matrix $M_{i j}=\tilde{M}_{i j} / \xi(0)$. Then Eq. (5) becomes

$$
\begin{aligned}
P_{N}\left(\vec{x}_{1}, \ldots, \vec{x}_{N}\right)= & \frac{(\operatorname{det} M)^{-1 / 2}}{(2 \pi T)^{N / 2}} \exp \left(-\frac{T}{2} \overrightarrow{1}^{T} M^{-1} \overrightarrow{1}\right) \\
& \cdot \int_{0}^{\infty} d \vec{\lambda} \exp \left(-\frac{1}{2 T} \vec{\lambda}^{T} M^{-1} \vec{\lambda}-\overrightarrow{1}^{T} M^{-1} \vec{\lambda}\right)
\end{aligned}
$$

To derive the asymptotic expansion of Eq. (6) for large $T$, we assume that

$$
\left(M^{-1} \overrightarrow{1}\right)_{i} \equiv \sum_{j=1}^{N} M_{i j}^{-1}>0 .
$$

This condition certainly holds at large separations $\left|\vec{x}_{i}-\vec{x}_{j}\right|$, where $\xi\left(\mid \vec{x}_{i}\right.$ $\left.-\vec{x}_{j} \mid\right) / \xi(0) \ll 1$. The $\vec{\lambda}$ integration can then be rewritten (using the convention that repeated indices are summed) as

Hence

$$
\left.\left\{\exp \left[-\frac{1}{2 T} \frac{\partial}{\partial J_{i}} M_{i k}^{-1} \frac{\partial}{\partial J_{k}}\right] \int_{0}^{\infty} d \vec{\lambda} \exp -\lambda_{j} J_{j}\right\}\right|_{J_{l}=\sum_{j=1}^{N} M_{i j}^{-1}} .
$$

$$
\begin{aligned}
P_{N}\left(\vec{x}_{1}, \ldots, \vec{x}_{N}\right)= & \frac{(\operatorname{det} M)^{-1 / 2}}{(2 \pi T)^{N / 2}} \exp \left[-\frac{T}{2} \overrightarrow{1}^{T} M^{-1} \overrightarrow{1}\right] \\
& \left.\cdot \exp \left(-\frac{1}{2 T} \frac{\partial}{\partial J_{i}} M_{i k}^{-1} \frac{\partial}{\partial J_{k}}\right)\left(\prod_{j=1}^{N} J_{j}\right)^{-1}\right|_{J_{j}=\left(M^{-1} 1\right)_{j}} .
\end{aligned}
$$

For large thresholds, the term $\exp \left(-(1 / 2 T) \vec{\nabla}_{J}^{T} M^{-1} \vec{\nabla}_{J}\right)$ can be expanded in a power series of inverse powers of $T$ yielding the asymptotic expansion. In the case $N=1$ the above becomes

$$
P_{1}=\frac{1}{\sqrt{2 \pi}} e^{-T / 2} \sum_{n=0}^{\infty}(-1)^{n}(2 n-1) ! ! T^{-(n+1 / 2)} .
$$

Equation (10) does not converge for any value of $T$, as is expected for an asymptotic expansion. The $N$-point correlations $\xi_{N}\left(\vec{x}_{1}, \ldots, \vec{x}_{N}\right)$, of places where $\varepsilon$ is above the threshold $T$, are related to the joint probabilities $P_{N}$ by

$$
1+\xi_{N}\left(\vec{x}_{1}, \ldots, \vec{x}_{N}\right)=P_{N}\left(\vec{x}_{1}, \ldots, \vec{x}_{N}\right) P_{1}^{-N} .
$$


Equations (9) and (10) then imply ${ }^{3}$

$$
\begin{aligned}
& \xi_{N}\left(\vec{x}_{1}, \ldots, \vec{x}_{N}\right)=-1+\left(\sum_{n=0}^{\infty}(-1)^{n}(2 n-1) ! ! T^{-n}\right)^{-N}(\operatorname{det} M)^{-1 / 2} \\
& \left.\cdot \exp \left(-\frac{T}{2} \sum_{i \neq j} M_{i j}^{-1}\right)\left\{\exp \left(-\frac{1}{2 T} M_{i j}^{-1} \frac{\partial}{\partial J_{i}} \frac{\partial}{\partial J_{j}}\right) \prod_{k=1}^{N} J_{k}^{-1}\right\}\right|_{J_{k}=\left(M^{-1}\right)_{k}} .
\end{aligned}
$$

The power series expansion of the terms in the brace brackets of Eq. (12) yields the asymptotic expansion for the correlation function $\xi_{N}\left(\vec{x}_{1}, \ldots, \vec{x}_{N}\right)$. To order $T^{-2}$ this expansion is

$$
\begin{aligned}
\xi_{N}\left(\vec{x}_{1}, \ldots, \vec{x}_{N}\right)= & -1+(\operatorname{det} M)^{-1 / 2} \exp \left(-\frac{T}{2} \sum_{i \neq j} M_{i j}^{-1}\right)\left(\prod_{m=1}^{N} J_{m}^{-1}\right) \\
& \cdot\left\{1+\frac{1}{T}\left(N-\sum_{i} \frac{M_{i i}^{-1}}{J_{i}^{2}}-\frac{1}{2} \sum_{i \neq j} \frac{M_{i j}^{-1}}{J_{i} J_{j}}\right)+\frac{1}{T^{2}}\left(\frac{N(N-5)}{2}\right.\right. \\
& -N \sum_{i} \frac{M_{i i}^{-1}}{J_{i}^{2}}-\frac{N}{2} \sum_{i \neq j} \frac{M_{i j}^{-1}}{J_{i} J_{j}}+3 \sum_{i} \frac{M_{i i}^{-1} M_{i i}^{-1}}{J_{i}^{4}}+\frac{1}{2} \sum_{i \neq k} \frac{M_{i i}^{-1} M_{k k}^{-1}}{J_{i}^{2} J_{k}^{2}} \\
& +\sum_{i \neq j} \frac{M_{i j}^{-1} M_{i j}^{-1}}{J_{i}^{2} J_{j}^{2}}+3 \sum_{i \neq k} \frac{M_{i i}^{-1} M_{i k}^{-1}}{J_{i}^{3} J_{k}}+\frac{1}{2} \sum_{i \neq k \neq l} \frac{M_{i i}^{-1} M_{k l}^{-1}}{J_{i}^{2} J_{k} J_{l}} \\
& \left.\left.+\sum_{i \neq l \neq j} \frac{M_{i l}^{-1} M_{i j}^{-1}}{J_{i}^{2} J_{l} J_{j}}+\frac{1}{8} \sum_{i \neq j \neq k \neq l} \frac{M_{i j}^{-1} M_{k l}^{-1}}{J_{i} J_{j} J_{k} J_{l}}\right)+O\left(1 / T^{3}\right)\right\},
\end{aligned}
$$

where $J_{i}=\sum_{j} M_{i j}^{-1}$. At large separations, where all the $\xi\left(\left|\vec{x}_{i}-\vec{x}_{j}\right|\right) / \xi(0)$ are small, an expansion in these quantities can also be made. From Eq. (13) we find that for the two point function,

$$
\begin{aligned}
1+\xi_{2}\left(\vec{x}_{1}-\vec{x}_{2}\right)= & \exp \left(T a /\left(1-a^{2}\right)\right)\left\{\left(1+2 a+\frac{3}{2} a^{2}\right)\right. \\
& \left.-T^{-1}\left(3 a+8 a^{2}\right)+T^{-2}\left(16 a+62 a^{2}\right)+O\left(T^{-3}, a^{3}\right)\right\},
\end{aligned}
$$

where $a=\xi\left(\left|\vec{x}_{1}-\vec{x}_{2}\right|\right) / \xi(0)$, and for the three point function

$$
\begin{aligned}
1+\xi_{3}\left(\vec{x}_{1}, \vec{x}_{2}, \vec{x}_{3}\right)= & \exp (T(A-B) /(1-C+2 D)) \\
& \cdot\left\{\left(1+2 A+3 B+\frac{3}{2} C\right)-T^{-1}(3 A+13 B+8 C)\right. \\
& \left.+T^{-2}(16 A+62 B+100 C)+O\left(T^{-3}, a_{i}^{3}\right)\right\},
\end{aligned}
$$

where $A=\sum_{i} a_{i}, \quad B=a_{1} a_{2}+a_{2} a_{3}+a_{3} a_{1}, \quad C=\sum_{i} a_{i}^{2}, \quad D=a_{1} a_{2} a_{3}, \quad$ and $a_{1}=$ $\xi\left(\vec{x}_{2}-\vec{x}_{3}\right) / \xi(0)$, etc. (In (14) and (15) one could expand the arguments of the exponentials in powers of $a_{i}$, but the number of terms which are important depends on how large $T a_{i}$ is.)

The leading contributions in (14) and (15) were previously derived in [4] where

3 An expansion for correlations of points where a Gaussian random field exceeds a high threshold was derived in ref. [5] using a different technique 
the factorization property

$$
1+\xi_{3}\left(\vec{x}_{1}, \vec{x}_{2}, \vec{x}_{3}\right)=\left(1+\xi_{2}\left(\vec{x}_{1}, \vec{x}_{2}\right)\right)\left(1+\xi_{2}\left(\vec{x}_{2}, \vec{x}_{3}\right)\right)\left(1+\xi_{2}\left(\vec{x}_{3}, \vec{x}_{1}\right)\right)
$$

was noted. Equations (14) and (15) show that this no longer holds for higher orders in $a_{i}$. However, (16) is still valid in higher orders of the $1 / T$ expansion as long as only leading order in $a_{i}$ is kept at each order of $1 / T$, including the exponent. (Although Eqs. (14) and (15) demonstrate this only to $O\left(T^{-2}\right)$, we conjecture that it is true to all orders.) Since the error from the exponent is the neglect of terms of order $\left(T a^{2}\right)$, whereas in the non-exponential factor a term of order $a / T$ is kept, this condition is sensible when $T a^{2} \ll a / T$, i.e., $\xi(x) / \xi(0) \ll 1 / T^{2}$. This is the regime in which factorization should continue to hold, even for moderately large $T$.

\section{High Peaks of a Gaussian Random Field}

The number density of local maxima in the Gaussian random field $\varepsilon(\vec{x})$ that occur at values of $\varepsilon$ greater than the threshold $t$ is given by

$$
n_{m}(\vec{x})=\int_{t}^{\infty} d m \sum_{i} \delta\left(\vec{x}-\vec{x}_{i}\right) \delta(\varepsilon(\vec{x})-m)
$$

where the sum runs over all positions $\vec{x}_{i}$ in $\mathbb{R}^{D}$ at which $\varepsilon\left(\vec{x}_{i}\right)$ is a local maximum. By Taylor expanding $\vec{\nabla} \varepsilon(\vec{x})$ near one of its local maxima, $\vec{x}_{i}$, it can be shown that

$$
\sum_{i} \delta\left(\vec{x}-\vec{x}_{i}\right)=\delta(\vec{\nabla} \varepsilon(\vec{x}))\left|\operatorname{det} \nabla_{a} \nabla_{b} \varepsilon(\vec{x})\right| .
$$

In addition to the vanishing of $\vec{\nabla} \varepsilon$, local maxima are characterized by the constraint that the symmetric $D \times D$ matrix with components $\omega^{a b}=\nabla_{a} \nabla_{b} \varepsilon(\vec{x})$ have negative eigenvalues. So the number density of local maxima is

$$
n_{m}(\vec{x})=\int_{t}^{\infty} d m \delta(\vec{\nabla} \varepsilon(\vec{x})) \delta(\varepsilon(\vec{x})-m) \int_{\mathscr{D}} d \omega \delta(\vec{\nabla} \vec{\nabla} \varepsilon(\vec{x})-\omega)(-1)^{D} \operatorname{det} \omega,
$$

where the region of integration $\mathscr{D}$ is over all negative definite $\omega$. (Hence $|\operatorname{det} \omega|=$ $\left.(-1)^{D} \operatorname{det} \omega\right)$. This is a complicated submanifold of $\mathbb{R}^{(1 / 2) D(D+1)}$. Note that $d \omega=\prod_{a \leqq b} d \omega^{a b}$, where $a, b=1, \ldots, D$. is

The probability density of finding $N$ peaks in the field $\varepsilon(\vec{x})$ at locations $\vec{x}_{1}, \ldots, \vec{x}_{N}$

$$
P_{N}\left(\vec{x}_{1}, \ldots, \vec{x}_{N}\right)=\int[d \varepsilon] P[\varepsilon] \prod_{i=1}^{N} n_{m}\left(\vec{x}_{i}\right),
$$

where $P[\varepsilon]$ is given by Eq. (2). The delta functions in $n_{m}$ can be written as integrals over exponentials so that after completing the square, Eq. (20) becomes a functional integral of the exponential of a quadratic form in $\varepsilon$. Performing the functional integration gives

$$
\begin{aligned}
P_{N}\left(\vec{x}_{1}, \ldots, \vec{x}_{N}\right)= & \left(\prod_{i=1}^{N} \int_{t}^{\infty} d m_{i} \int_{\mathscr{D}} d \omega_{i} \int \frac{d \vec{k}_{i}}{(2 \pi)^{d}}(-1)^{D} \operatorname{det} \omega_{i}\right) \\
& \cdot \exp \left(-\frac{1}{2} \sum_{A, B, j, k} k_{j}^{A} \Lambda_{j k}^{A B} k_{k}^{B}-i \sum_{A, j} k_{j}^{A} N_{j}^{A}\right) .
\end{aligned}
$$


In Eq. (21) the $\omega_{j}, j=1 \ldots N$, are $D \times D$ symmetric matrices with components $\left(\omega_{j}\right)^{a b}$, $a, b=1, \ldots, D$. The $\vec{N}_{j}$ are vectors with $d=1+D+\frac{1}{2} D(D+1)$ components, $N_{j}^{A}$, $A=1, \ldots, d$. Explicitly

Finally,

$$
\begin{aligned}
\vec{N}_{j} & \left.=\left(\begin{array}{l}
m_{j} \\
0 \\
\vdots \\
0 \\
\left(\omega_{j}\right)^{a b}, a=1, \ldots, D ; b \leqq a
\end{array}\right)\right\} \frac{1}{2} D(D+1) \\
& \equiv\left(\begin{array}{l}
\vec{N}_{j} \\
\omega_{j}
\end{array}\right)
\end{aligned}
$$

$$
\Lambda_{j k}^{A B}=D_{j}^{A} D_{k}^{B} \xi\left(\left|\vec{x}_{j}-\vec{x}_{k}\right|\right),
$$

where

$$
\vec{D}_{j}=\left(\begin{array}{l}
1 \\
\frac{\partial}{\partial x_{j}^{a}} \\
\frac{\partial^{2}}{\partial x_{j}^{a} \partial x_{j}^{b}}, a=1, \ldots, D ; b \leqq a
\end{array}\right) \begin{aligned}
& \} 1 \\
& \} \\
& \frac{1}{2} D(D+1)
\end{aligned}
$$

For fixed subscripts (1.c. indices labeling the $N$ points $\left.\vec{x}_{i}\right) \vec{k}_{i}$ and $\vec{D}_{i}$ are $d$-vectors and $\Lambda_{j k}$ is a $d \times d$ symmetric matrix. The appendix discusses the form of $\Lambda$ in greater detail.

Performing the $k$ integrations gives

$$
P_{N}\left(\vec{x}_{1}, \ldots, \vec{x}_{N}\right)=\left(\prod_{j=1}^{N} \int_{t}^{\infty} d m_{j} \int_{\mathscr{D}} d \omega_{j}(-1)^{D} \operatorname{det} \omega_{j}\right) \frac{\exp \left(-\frac{1}{2} \vec{N}^{T} \Lambda^{-1} \vec{N}\right)}{\left[(2 \pi)^{N d} \operatorname{det} \Lambda\right]^{1 / 2}} .
$$

In Eq. (24) $\vec{N}$ is a $N d$-vector with components $N_{j}^{A}$ and $\Lambda$ is a symmetric $N d \times N d$ matrix with components $\Lambda_{j k}^{A B}$. It proves convenient to decompose $\Lambda$ into submatrices corresponding to its upper case indices as follows

$$
\Lambda=(\underbrace{A}_{(D+1) N} \underbrace{\frac{1}{C^{T}}}_{\frac{1}{2} D(D+1) / N}+\underbrace{\frac{C}{B}}-)_{3 \frac{1}{2} D(D+1) N}^{(D+1) N}
$$

where, again, the $(D+1) N$ components reflect the $\vec{\nabla} \varepsilon=0$ and $\varepsilon \geqq t$ constraints and the $\frac{1}{2} D(D+1) N$ components reflect the negativity of $\vec{\nabla} \vec{\nabla} \varepsilon$ constraint. Decomposing $\Lambda^{-1}$ in an analogous fashion,

$$
\Lambda^{-1}=\left(\begin{array}{ll}
X & Z \\
Z^{T} & Y
\end{array}\right)
$$


it is straightforward to show that

$$
X=A^{-1}+Z Y^{-1} Z^{T} .
$$

Therefore the argument of the exponential in Eq. (24) is

where

$$
\vec{N}^{T} \Lambda^{-1} \vec{N}=\overrightarrow{\bar{N}}^{T} A^{-1} \overrightarrow{\bar{N}}+(\vec{\omega}-\vec{G})^{T} Y(\vec{\omega}-\vec{G}),
$$

$$
\vec{G}=-Y^{-1} Z^{T} \vec{N}
$$

Here $\vec{\omega}$ is the vector whose $\frac{1}{2} N D(D+1)$ components are the independent components of the $N$ symmetric $D \times D$ matrices $\omega_{j}$. Similarly, $\vec{N}$ is a $N(D+1)$ dimensional vector whose components are $\bar{N}_{j}^{A}$. Using $\Lambda \Lambda^{-1}=1$, one deduces that $Y^{-1} Z^{T}=-C^{T} A^{-1}$. So $\vec{G}$ has components

$$
G_{j}^{a b}=\left(C^{T}\right)_{j, k}^{a b, c}\left(A^{-1}\right)_{k l}^{c d} \bar{N}_{l}^{d},
$$

where $a, b=1, \ldots, D$ with $b \leqq a ; c, d=1, \ldots, D+1$ and $j, k, l=1, \ldots, N$. Because $\bar{N}_{l}^{d}=\delta_{1}^{d} m_{l}$, with the further definition

$$
\left(\bar{A}^{-1}\right)_{i j}=\left(A^{-1}\right)_{i j}^{11},
$$

our expression for the joint probability density that peaks are located at positions $\vec{x}_{1}, \ldots, \vec{x}_{N}$ becomes

$$
\begin{aligned}
P_{N}\left(\vec{x}_{1}, \ldots, \vec{x}_{N}\right)= & {\left[(2 \pi)^{N d} \operatorname{det} \Lambda\right]^{-1 / 2}\left(\prod_{j=1}^{N} \int_{t}^{\infty} d m_{j}\right) \exp \left(-\frac{1}{2} \vec{m}^{T} \bar{A}^{-1} \vec{m}\right) } \\
& \cdot\left(\prod_{k=1}^{N} \int_{\mathscr{D}} d \omega_{k}(-)^{D} \operatorname{det} \omega_{k}\right) \exp \left(-\frac{1}{2}(\vec{\omega}-\vec{G})^{T} Y(\vec{\omega}-\vec{G})\right) .
\end{aligned}
$$

The integration region $\mathscr{D}$ is quite complicated. However for high thresholds extending the region $\mathscr{D}$ to $\mathbb{R}^{(1 / 2) D(D+1)}$ induces only small errors. So we shall approximate $P_{N}$ by $P_{N}^{\prime}$, where

$$
\begin{aligned}
P_{N}^{\prime}\left(\vec{x}_{1}, \ldots, \vec{x}_{N}\right)= & {\left[(2 \pi)^{N d} \operatorname{det} \Lambda\right]^{-1 / 2} \prod_{j=1}^{N} \int_{t}^{\infty} d m_{j} } \\
& \cdot \exp \left(-\frac{1}{2} \vec{m}^{T} \bar{A}^{-1} \vec{m}\right) I_{N}^{\prime}\left[\mathbb{R}^{(1 / 2) N D(D+1)}\right] \\
I_{N}^{\prime}\left[\mathscr{A}^{N}\right]= & (-1)^{N D} \int_{\mathscr{A}^{N}} \prod_{k=1}^{N} d \omega_{k} \operatorname{det} \omega_{k} \exp \left(-\frac{1}{2}(\vec{\omega}-\vec{G})^{T} Y(\vec{\omega}-\vec{G})\right)
\end{aligned}
$$

and $\mathscr{A}^{N}=\mathscr{A} \times \mathscr{A} \times \cdots \times \mathscr{A}$. ( $\vec{G}$ is a linear function of $\vec{m}$ - see Eq. (30).) Note that $P_{N}^{\prime}$ includes contributions from minima and saddle points, as well as maxima. The error made by using $P_{N}^{\prime}$ instead of $P_{N}$ will be estimated in the next section. Roughly speaking, the relative errors are of order $\exp \left(-T \xi(0)^{2} / \xi(x)^{2}\right) / \exp (-T)$ and $\exp (-T)$; see Eq. (104).

Expressing the determinants of $\omega_{j}$ as integrals over Grassmann variables $\eta$ and $\bar{\eta}$ [6],

$$
\operatorname{det} \omega_{j}=\int \prod_{a=1}^{D} d \eta_{j}^{a} d \bar{\eta}_{j}^{a} \exp \left(\bar{\eta}_{j}^{a}\left(\omega_{j}\right)^{a b} \eta_{j}^{b}\right)
$$


allows us to easily perform the $\omega$ integrations. (Note that, in contrast with $a$ and $b$, the $j$ indices are not summed over in the exponent.) This gives

$$
\begin{aligned}
I_{N}^{\prime}\left[\mathbb{R}^{(1 / 2) N D(D+1)}\right]= & \left(\prod_{k=1}^{N} \int\left(\prod_{a=1}^{D} d \eta_{k}^{a} d \bar{\eta}_{k}^{a}\right)\right) \\
& \cdot \exp \left(\sum_{j} \bar{\eta}_{j}^{a} G_{j}^{a b} \eta_{j}^{b}+\frac{1}{2} \sum_{k, l} \bar{\eta}_{k}^{a} \eta_{k}^{b}\left(Y^{-1}\right)_{k, l}^{a b, c d} \bar{\eta}_{l}^{c} \eta_{l}^{d}\right) .
\end{aligned}
$$

The asymptotic series in $T^{-1}$ for $P_{N}^{\prime}$ is derived by expanding the exponential of the quartic term in the Grassmann variables in a power series, performing the Grassmann integrations, and then performing the $m_{j}$ integrations using the technique introduced in Sect. II. The Grassmann integrations are performed using the identity

$$
\int \prod_{i} d \eta_{i} d \bar{\eta}_{i}\left(\bar{\eta}_{i_{1}} \eta_{j_{1}}\right) \ldots\left(\bar{\eta}_{i_{m}} \eta_{j_{m}}\right) e^{\bar{\eta}_{j} A_{j k} \eta_{k}}=\operatorname{det} A \sum_{P \in S_{m}} \delta_{P} A_{i_{1} P\left(j_{1}\right)}^{-1} \ldots A_{i_{m} P\left(j_{m}\right)}^{-1},
$$

where $S_{m}$ are the permutations of the $m$ indices $j_{1} \ldots j_{m}$ and $\delta_{P}$ is the sign of the permutation. So our expansion in $T^{-1}$ of $I_{N}^{\prime}$ becomes

$$
\begin{aligned}
I_{N}^{\prime}\left[\mathbb{R}^{(1 / 2) N D(D+1)}\right]= & (-)^{N D}(2 \pi)^{N D(D+1) / 4}(\operatorname{det} Y)^{-1 / 2} \prod_{j=1}^{N} \operatorname{det} G_{j} \\
& \cdot\left\{1+\frac{1}{2}\left(Y^{-1}\right)_{k l}^{a b, c d}\left[\left(G_{k}^{-1}\right)^{a b}\left(G_{l}^{-1}\right)^{c d}\right.\right. \\
& \left.\left.-\left(G_{k}^{-1}\right)^{a d}\left(G_{k}^{-1}\right)^{c b} \delta_{k l}\right]+O\left(G^{-1}\right)^{4}\right\} .
\end{aligned}
$$

Let us define a matrix $\varepsilon$ such that

$$
G_{j}^{a b}=-\alpha \delta^{a b} m_{j}+\varepsilon_{j k}^{a b} m_{k},
$$

where

$$
\alpha=-\nabla^{2} \xi(0) / D \xi(0)>0
$$

hence

$$
\left(G_{j}^{-1}\right)^{a b}=-\frac{\delta^{a b}}{\alpha m_{j}}-\frac{\varepsilon_{j k}^{a b} m_{k}}{\left(\alpha m_{j}\right)^{2}}+O\left(\varepsilon^{2}\right) .
$$

For large separations, $\varepsilon$ can be expanded in powers of $\xi(x) / \xi(0)$. From Eq. (30) we find that ${ }^{4}$

$$
\varepsilon_{j k}^{a b}=\left(1-\delta_{j k}\right)\left(\alpha \delta^{a b}+\nabla_{a} \nabla_{b}\right) \hat{\xi}_{j k}+O\left(\hat{\xi}^{2}\right),
$$

and we have introduced

$$
\widehat{\xi}_{j k} \equiv \xi_{j k} / \xi(0) \equiv \xi\left(\left|\vec{x}_{j}-\vec{x}_{k}\right|\right) / \xi(0), \quad j \neq k
$$

So we have ${ }^{5}$

4 Note that throughout, $O\left(\hat{\xi}^{2}\right)$ means $O\left(\hat{\xi}^{2},(\nabla \hat{\xi})^{2}, \hat{\xi} \nabla^{2} \hat{\xi}\right.$, etc. $)$

5 In Eq. (42) we omit a term of the form $\sum_{k \neq 1}\left(Y^{-1}\right)_{k l} \varepsilon m / m^{3}$ because $\left(Y^{-1}\right)_{k l}$ is $O\left(\xi_{k l}\right)$ for $k \neq l$; see Eq. (45) 


$$
\begin{aligned}
& I_{N}^{\prime}\left[\mathbb{R}^{(1 / 2) N D(D+1)}\right] \\
&=(2 \pi)^{(1 / 4) N D(D+1)}(\operatorname{det} Y)^{-1 / 2} \prod_{j=1}^{N}\left(\alpha m_{j}\right)^{D}\left(1-\alpha^{-1} \sum_{j, l} \varepsilon_{j l}^{a a} \frac{m_{l}}{m_{j}}+O\left(\hat{\xi}_{i j}^{2}\right)\right) \\
& \cdot\left\{1+\frac{\alpha^{-2}}{2} \sum_{k}\left[\left(Y^{-1}\right)_{k, k}^{a a, b b}-\left(Y^{-1}\right)_{k, k}^{a b, b a}\right] \frac{1}{m_{k}^{2}}\right. \\
&+\frac{\alpha^{-2}}{2} \sum_{k \neq l}\left(Y^{-1}\right)_{k, l}^{a a, b b} \frac{1}{m_{k} m_{l}} \\
&\left.+\alpha^{-3} \sum_{k, l}\left[\left(Y^{-1}\right)_{k, k}^{a a, b c}-\left(Y^{-1}\right)_{k, k}^{a b, c a}\right] \frac{\varepsilon_{k l}^{b c} m_{l}}{m_{k}^{3}}+O\left(\xi_{i j}^{2}, m^{-4}\right)\right\} .
\end{aligned}
$$

The terms involving $Y^{-1}$ can be evaluated in terms of $\xi_{i j}$ and its derivatives using the identity

$$
Y^{-1}=B-C^{T} A^{-1} C,
$$

which follows from expanding $\Lambda \Lambda^{-1}=1$ in terms of the submatrices (Eqs. (25) and (26)) and eliminating $Z$ from the resulting equations

$$
C^{T} Z+B Y=1 \text { and } A Z+C Y=0 .
$$

Using Eq. (43) and the results in the Appendix, we find that

$$
\left(Y^{-1}\right)_{i, j}^{a b, c d}=\left[\nabla_{a} \nabla_{b} \nabla_{c} \nabla_{d}+\alpha\left(\delta^{a b} \nabla_{c} \nabla_{d}+\delta^{c d} \nabla_{a} \nabla_{b}\right)+\alpha^{2} \delta^{a b} \delta^{c d}\right] \xi_{i j}+O\left(\xi_{i j}^{2}\right),
$$

which implies that

$$
\begin{gathered}
\left(Y^{-1}\right)_{k, k}^{a a, b b}-\left(Y^{-1}\right)_{k, k}^{a b, b a}=-D(D-1) \alpha^{2} \xi(0), \\
\left(Y^{-1}\right)_{k, k}^{a a, b c}-\left(Y^{-1}\right)_{k, k}^{a b, c a}=-\delta^{b c}(D-1) \alpha^{2} \xi(0) .
\end{gathered}
$$

Using Eqs. (40), (45) and (46), $I_{N}^{\prime}$ becomes

$$
\begin{aligned}
I_{N}^{\prime}\left[\mathbb{R}^{(1 / 2) N D(D+1)}\right]= & (2 \pi)^{(1 / 4) N D(D+1)}(\operatorname{det} Y)^{-1 / 2} \prod_{j=1}^{N}\left(\alpha m_{j}\right)^{D}\left(1-\sum_{j \neq l} \frac{m_{l}}{m_{j}}\left(D+\frac{\nabla^{2}}{\alpha}\right) \hat{\xi}_{j l}\right. \\
& \left.+O\left(\hat{\xi}_{i j}^{2}\right)\right)\left\{1-\sum_{k} \frac{D(D-1)}{2 m_{k}^{2}} \xi(0)-\sum_{k \neq l} \frac{1}{2 m_{k} m_{l}}\left(D+\frac{\nabla^{2}}{\alpha}\right)^{2} \xi_{k l}\right. \\
& \left.-\sum_{k \neq l} \frac{m_{l}}{m_{k}^{3}}(D-1)\left(D+\frac{\nabla^{2}}{\alpha}\right) \xi_{k l}+O\left(\xi_{i j}^{2}, m^{-4}\right)\right\} .
\end{aligned}
$$

After substituting this result into Eq. (33) for $P_{N}^{\prime}$, the $m_{j}$ integrations can be performed by the same technique as in Sect. II, giving

$$
\begin{aligned}
P_{N}^{\prime}\left(\vec{x}_{1}, \ldots, \vec{x}_{N}\right)= & {\left[(2 \pi)^{N d} \operatorname{det} \Lambda\right]^{-1 / 2}(2 \pi)^{N D(D+1) / 4}(\operatorname{det} Y)^{-1 / 2} \alpha^{N D} t^{N(D-1)} } \\
& \cdot \exp \left(-\frac{1}{2} t^{2} \overrightarrow{1}^{T} \bar{A}^{-1} \overrightarrow{1}\right)\left(\prod_{i} J_{i}^{-1}\right)\left(1-\sum_{j \neq l} \hat{O} \hat{\xi}_{j l}+O\left(\hat{\xi}_{i j}^{2}\right)\right) \\
& \cdot\left\{1+\frac{D}{t^{2}} \sum_{i} J_{i}^{-1}-\frac{N D(D-1) \xi(0)}{-2 t^{2}}+\frac{1}{2 t^{2}}\left(\sum_{k} \frac{2 \bar{A}_{k k}^{-1}}{J_{k}^{2}}+\sum_{k \neq l} \frac{\bar{A}_{k l}^{-1}}{J_{k} J_{l}}\right)\right. \\
& \left.-\frac{1}{2 t^{2}} \sum_{k \neq l}\left(\hat{O}^{2}+2(D-1) \hat{O}\right) \hat{\xi}_{k l}+O\left(\hat{\xi}_{i j}^{2}, t^{-4}\right)\right\},
\end{aligned}
$$


where $J_{i}=\sum_{j} \bar{A}_{i j}^{-1}$ and $\hat{O}=D+\alpha^{-1} \nabla^{2}$. To evaluate the determinants in (48), we note from (44) that

$$
\left(\begin{array}{ll}
A & C \\
C^{T} & B
\end{array}\right)\left(\begin{array}{ll}
1 & Z \\
0 & Y
\end{array}\right)=\left(\begin{array}{ll}
A & 0 \\
C^{T} & 1
\end{array}\right)
$$

hence

$$
\operatorname{det} \Lambda \operatorname{det} Y=\operatorname{det} A \text {. }
$$

Since the diagonal part of $A$ is $N$ blocks of $\xi(0) \operatorname{diag}(1, \alpha, \ldots, \alpha)$ and the off diagonal elements are $O\left(\xi_{i j}\right)$,

$$
\sqrt{\operatorname{det} A}=\xi(0)^{N(D+1) / 2} \alpha^{N D / 2}\left(1+O\left(\xi_{i j}^{2}\right)\right) .
$$

Also needed is the large distance expansion of $\bar{A}^{-1}$,

$$
\bar{A}_{i j}^{-1}=\frac{\delta_{i j}}{\xi(0)}-\left(1-\delta_{i j}\right) \frac{\xi_{i j}}{(\xi(0))^{2}}+O\left(\xi_{i j}^{2}\right)
$$

Equations (48)-(52) imply that

$$
\begin{aligned}
P_{N}^{\prime}\left(\vec{x}_{1}, \ldots, \vec{x}_{N}\right)= & (2 \pi)^{-(1 / 2) N(D+1)} \alpha^{(1 / 2) N D} \xi(0)^{-(1 / 2) N(D-1)} t^{N(D-1)} \\
& \cdot \exp \left(-\frac{1}{2} t^{2} \overrightarrow{1}^{T} \bar{A}^{-1} \overrightarrow{1}\right)\{(1-2(D-1) x-2 y) \\
& +\frac{\xi(0)}{t^{2}}\left(N(D-1)\left(1-\frac{1}{2} D\right)+x(D-1)[N(D-1)(D-2)-(D-3)]\right. \\
& \left.+y[N(D-1)(D-2)+2]+z)+O\left(\xi_{i j}^{2}, t^{-4}\right)\right\}
\end{aligned}
$$

where

$$
x=\sum_{i<j} \hat{\xi}_{i j}, \quad y=\sum_{i<j} \alpha^{-1} \nabla^{2} \hat{\xi}_{i j}, \quad z=\sum_{i<j} \alpha^{-2} \nabla^{4} \hat{\xi}_{i j} .
$$

The number density of peaks above the high threshold $t$ is $P_{1}^{\prime}$, which from (53) is

$$
\begin{aligned}
P_{1}^{\prime}= & \frac{\alpha^{D / 2} t^{D-1}}{(2 \pi)^{(D+1) / 2} \xi(0)^{(D-1) / 2}} \\
& \cdot \exp \left(-t^{2} / 2 \xi(0)\right)\left\{1+(D-1)\left(1-\frac{1}{2} D\right) \frac{\xi(0)}{t^{2}}+O\left(t^{-4}\right)\right\} .
\end{aligned}
$$

This generalizes previous results which omitted the subleading term [7] or derived it in only the $D=3$ case $[3,8]$.

The $N$-point correlations of the peaks, $\xi_{N}$, are defined similarly to Eq. (11),

$$
1+\xi_{N}\left(\vec{x}_{1}, \ldots, \vec{x}_{N}\right)=P_{N}^{\prime}\left(\vec{x}_{1}, \ldots, \vec{x}_{N}\right) /\left(P_{1}^{\prime}\right)^{N},
$$

which according to Eqs. (53) and (55) is

$$
1+\xi_{N}\left(\vec{x}_{1}, \ldots, \vec{x}_{N}\right)=\exp \left(-\frac{t^{2}}{2}\left(\overrightarrow{1}^{T} \bar{A}^{-1} \overrightarrow{1}-N / \xi(0)\right)\right)
$$




$$
\begin{aligned}
& \cdot\left\{[1-2(D-1) x-2 y]+\frac{\xi(0)}{t^{2}}[-(D-1)(D-3) x+2 y+z]\right. \\
& \left.+O\left(\xi_{i j}^{2}, t^{-4}\right)\right\} .
\end{aligned}
$$

Equations (55) and (57) are the main results of this paper. Using

$$
\bar{A}_{i j}^{-1}=\frac{\delta_{i j}}{\xi(0)}-\left(1-\delta_{i j}\right) \frac{\xi_{i j}}{\xi(0)^{2}}+\frac{1}{\xi(0)^{3}} \sum_{l \neq l, j}\left[\xi_{i l} \xi_{l j}+\frac{1}{\alpha} \vec{\nabla}_{l} \xi_{i l} \cdot \vec{\nabla}_{l} \xi_{l j}\right]+\cdots,
$$

the argument of the exponent can be expanded in powers of the correlations $\xi_{i j}$ which are small at large separations $\left|\vec{x}_{i}-\vec{x}_{j}\right|$. For example with $D=3$, the two and three point peak correlations are approximately

$$
\begin{aligned}
& 1+\xi_{2}\left(\vec{x}_{1}, \vec{x}_{2}\right)=\left\{\left(1-4 \frac{\xi_{12}}{\xi(0)}+6 \frac{\nabla^{2} \xi_{12}}{\nabla^{2} \xi(0)}\right)\right. \\
&\left.+T^{-1}\left(-6 \frac{\nabla^{2} \xi_{12}}{\nabla^{2} \xi(0)}+9 \xi(0) \frac{\nabla^{4} \xi_{12}}{\left(\nabla^{2} \xi(0)\right)^{2}}\right)+O\left(\xi_{12}^{2}, T^{-2}\right)\right\} \\
& \cdot \exp \left\{T\left(\hat{\xi}_{12}-\hat{\xi}_{12}^{2}+3 \frac{\left(\vec{\nabla}_{12}\right)^{2}}{\nabla^{2} \xi(0) / \xi(0)}+O\left(\hat{\xi}_{12}^{3}\right)\right)\right\}, \\
& 1+\xi_{3}\left(\vec{x}_{1}, \vec{x}_{2}, \vec{x}_{3}\right)=\left[1+\xi_{2}\left(\vec{x}_{1}, \vec{x}_{2}\right)\right]\left[1+\xi_{2}\left(\vec{x}_{2}, \vec{x}_{3}\right)\right]\left[1+\xi_{2}\left(\vec{x}_{3}, \vec{x}_{1}\right)\right] \\
& \cdot \exp \left\{T\left(-\hat{\xi}_{12} \hat{\xi}_{23}+3 \frac{\vec{\nabla}_{2} \hat{\xi}_{12} \cdot \vec{\nabla}_{2} \hat{\xi}_{23}}{\nabla^{2} \xi(0) / \xi(0)}+\text { perm. }+O\left(\hat{\xi}^{3}\right)\right)\right\} \\
&+ O\left(\hat{\xi}^{2}\right),
\end{aligned}
$$

where "perm." denotes the cyclic permutations of the indices, and $T=t^{2} / \xi(0)$. Equation (60) has appeared previously, although its derivation was incorrect in ref. [9].

Note that to leading order in $T$ and $\hat{\xi}$ the correlations of peaks are the same as those for points above threshold found in Sect. II (Eqs. (14) and (15)). Equation (60) shows that the factorization property

$$
1+\xi_{N}\left(\vec{x}_{1}, \ldots, \vec{x}_{N}\right)=\prod_{i<j}\left(1+\xi_{2}\left(\vec{x}_{i}, \vec{x}_{j}\right)\right)
$$

that holds to leading order in $\hat{\xi}$ (for each order of $T^{-1}$ ) does not hold when terms of order $\hat{\xi}^{2}$ are kept. The situation is therefore just the same as for points above threshold, so that the comments following Eq. (16) apply here as well.

\section{IV. $P_{N}^{\prime}$ Versus $P_{N}$}

In Sect. III it was shown that the joint probability density for local maxima, exceeding height $t$, to be located at the positions $\vec{x}_{1}, \ldots, \vec{x}_{N}$ is given by

$$
P_{N}\left(\vec{x}_{1}, \ldots, \vec{x}_{N}\right)=k_{N} \prod_{j=1}^{N} \int_{t}^{\infty} d m_{j} \exp \left(-\frac{1}{2} \vec{m}^{T} \bar{A}^{-1} \vec{m}\right) I_{N}^{\prime}\left[\mathscr{D}^{N}\right],
$$


where $k_{N}$ is the prefactor in Eq. (32) and $I_{N}^{\prime}$ is given in Eq. (33). Recall that the $\omega_{k}$ integration region $\mathscr{D}$ is over symmetric negative definite $D \times D$ matrices, to enforce the peak constraint. This is a complicated subspace of $\mathbb{R}^{(1 / 2) D(D+1)}$, but we shall prove that for high thresholds $t \gg \sqrt{\xi(0)}$, and large separations $\left|\vec{x}_{i}-\vec{x}_{j}\right| \gg \sqrt{1 / \alpha}, P_{N}$ can be approximated by

$$
P_{N}^{\prime}\left(\vec{x}_{1}, \ldots, \vec{x}_{N}\right)=k_{N} \prod_{j=1}^{N} \int_{t}^{\infty} d m_{j} \exp \left(-\frac{1}{2} \vec{m}^{T} \bar{A}^{-1} \vec{m}\right) I_{N}^{\prime}\left[\mathbb{R}^{(1 / 2) N D(D+1)}\right] .
$$

$P_{N}^{\prime}$ differs from $P_{N}$ because the range of integration has been extended from $\mathscr{D}$ to $\mathbb{R}^{(1 / 2) D(D+1)}$. In the theorem of this section we show that the error in replacing $\mathscr{D}$ by $\mathbb{R}^{(1 / 2) D(D+1)}$ for large separations and threshold is negligible compared to the terms kept in Sect. III. Prior to the theorem it will be helpful to have the following lemmas.

Lemma 1. Let $\mathscr{A}$ be the region of $\omega$-space defined by $\omega_{a a} \leqq 0$ for $a=1, \ldots, D$ and $\left|\omega_{a b}\right| \leqq \eta(D) \sqrt{\omega_{a a} \omega_{b b}}$ for $a<b=2, \ldots, D$, with $\eta(D)=(D !)^{-1 / 2}$. Then $\mathscr{A} \subset \mathscr{D}$ (where $\mathscr{D}$ is the space of symmetric negative definite $D \times D$ matrices).

Proof. Let $\omega_{a b}^{(m)}$ be the matrix obtained from $\omega_{a b}$ by restricting the indices $a$ and $b$ by $a, b \leqq m \leqq D$, and let $\Delta_{m}=\operatorname{det} \omega^{(m)}$. A standard result from linear algebra [10] is that $\omega$ is negative definite if and only if $\forall_{m}(-)^{m} \Delta_{m}>0$. Write

$$
\begin{gathered}
\Delta_{m}=\omega_{11} \ldots \omega_{m m}+B \\
B=\frac{1}{m !} \varepsilon_{a_{1} \ldots a_{m}} \varepsilon_{b_{1} \ldots b_{m}} \omega_{a_{1} b_{1}} \ldots \omega_{a_{m} b_{m}}-\omega_{11} \ldots \omega_{m m} .
\end{gathered}
$$

Hence $B$ is the sum of $m !-1$ terms $b_{\alpha}$. For $m>1$ we note that each $b_{\alpha}$ has at most $m-2$ diagonal elements of $\omega$. This implies that

and so

$$
\left|b_{\alpha}\right| \leqq \eta^{2}\left|\omega_{11} \ldots \omega_{m m}\right|
$$

$$
|B| \leqq(m !-1) \eta^{2}\left|\omega_{11} \ldots \omega_{m m}\right|=\frac{m !-1}{D !}\left|\omega_{11} \ldots \omega_{m m}\right|
$$

But $m \leqq D$, so $(-1)^{m} \omega_{11} \ldots \omega_{m m}=\left|\omega_{11} \ldots \omega_{m m}\right|>|B|$, and $(-1)^{m} \Delta_{m}>0$ for $m>1$. For $m=1,-\Delta_{1}>0$ since $\omega_{11}<0$, completing the proof.

Lemma 2. Consider the one-dimensional integral $I[\mathscr{E}]$,

$$
I[\mathscr{E}]=\int_{\mathscr{E}} d \omega\left|\omega^{n}\right| \exp \left(-\frac{1}{2} y(\omega-g)^{2}\right),
$$

where $n=0,1, \ldots, y>0$, and the region of integration $\mathscr{E}$ is such that

Then

$$
r=\inf _{\omega \in \mathscr{E}}|\omega-g|>0
$$

$$
I[\mathscr{E}]<\frac{1}{r y} e^{-(1 / 2) r^{2} y} Q_{n}\left(r,|g|, y^{-1 / 2}\right),
$$

where $Q_{n}$ is a homogeneous multinomial of degree $n$ in the arguments. 
Proof. Since the integrand is positive,

$$
\begin{aligned}
I[\mathscr{E}] & <I[(-\infty, g-r] \cup[g+r, \infty)] \\
& =\left(\int_{-\infty}^{-r}+\int_{r}^{\infty}\right) d \omega|\omega+g|^{n} e^{-(1 / 2) y \omega^{2}} \\
& \leqq 2 \int_{r}^{\infty} d \omega(\omega+|g|)^{n} e^{-(1 / 2) y \omega^{2}} \\
& =2 \sum_{j=0}^{n}\left(\begin{array}{l}
n \\
j
\end{array}\right)|g|^{n-j} \int_{r}^{\infty} d \omega \omega^{j} e^{-(1 / 2) y \omega^{2}} .
\end{aligned}
$$

Integration by parts and the inequality

$$
\int_{r}^{\infty} d \omega e^{-(1 / 2) y \omega^{2}}<\frac{1}{r y} e^{-(1 / 2) r^{2} y}
$$

gives the desired result.

The theorem we now present is essentially a generalization of Lemma 2 to the more complicated integral $I_{N}^{\prime}$, with the correspondences

$$
\begin{aligned}
\mathscr{E} & \rightarrow \mathbb{R}^{(1 / 2) N D(D+1)}-\mathscr{D}^{N}, \\
\omega & \rightarrow \omega_{i}^{a b}, \\
y & \rightarrow Y_{i, j}^{a b, c d}, \\
g & \rightarrow G_{j}^{a b}, \\
r & \rightarrow O(t) .
\end{aligned}
$$

The basic idea is that, since $G_{j}^{a b}=-\alpha \delta^{a b} m_{j}+\varepsilon_{j k}^{a b} m_{k}$ (Eq. (39)), $\omega_{j}^{a b}=G_{j}^{a b}$ will lie within the integration region $\mathscr{D}$ as long as $\varepsilon_{j k}^{a b} m_{k}$ is not too large, i.e. $m_{i} \leqq(\alpha /|\varepsilon|) t$. (This is because $-\alpha \delta^{a b}$ is a negative definite matrix.) Then by the higher dimensional analog of Lemma 2 , the error in extending $\mathscr{D}$ to $\mathbb{R}^{(1 / 2) D(D+1)}$ will be exponentially small in the threshold. On the other hand, if $m_{i} \geqq(\alpha /|\varepsilon|) t, \omega=G$ may lie outside $\mathscr{D}$, in which case the above strategy cannot be used. But these contributions to $P_{N}$ are suppressed by the factor $\exp \left(-\frac{1}{2} \vec{m}^{T} \bar{A}^{-1} \vec{m}\right)$ when the integration over $\vec{m}$ is performed.

Theorem. Assume $Y$ and $\bar{A}^{-1}$ are positive definite (which will be justified presently); then

$$
\begin{gathered}
\left|P_{N}\left(\vec{x}_{1}, \ldots, \vec{x}_{N}\right)-P_{N}^{\prime}\left(\vec{x}_{1}, \ldots, \vec{x}_{N}\right)\right|<\left[(2 \pi)^{N D} \operatorname{det} A\right]^{-1 / 2} t^{-N}\left(\operatorname{det} \bar{A}^{-1}\right)^{-1} \\
\cdot\left\{\frac{\exp \left(-\frac{1}{2} r^{2} \operatorname{tr} Y-\frac{1}{2} t^{2} \operatorname{tr} \bar{A}^{-1}\right)}{r^{(1 / 2) N D(D+1)}(\operatorname{det} Y)^{1 / 2}} Q_{N D}^{(1)}(\alpha t)+\frac{\exp \left(-\frac{1}{2} \bar{m}^{2} \operatorname{tr} \bar{A}^{-1}\right)}{\left(\frac{1}{2 N}\left(\frac{\alpha}{\varepsilon}\right) \frac{\eta}{1+\eta}\right)^{N}} Q_{N D}^{(2)}(\alpha \bar{m})\right\},
\end{gathered}
$$

where

$$
r=\frac{1}{2}\left(\frac{1}{2} N D(D+1)\right)^{-1 / 2}\left(\frac{\eta}{1+\eta}\right) \alpha t,
$$




$$
\begin{aligned}
\bar{m} & =\frac{1}{2 N}\left(\frac{\alpha}{\varepsilon}\right)\left(\frac{\eta}{1+\eta}\right) t, \\
\varepsilon & =\max _{a, b, i, j}\left\{\left|\varepsilon_{i j}^{a b}\right|\right\}, \quad \eta=(D !)^{-1 / 2},
\end{aligned}
$$

and $Q_{N D}^{(1,2)}$ are polynominals of degree $N D$ whose coefficients are well-behaved ${ }^{6}$ functions of $Y$, and $\bar{A}^{-1}$, (For the explicit form of $\varepsilon_{i j}^{a b}$, see Eq. (40).)

Proof. Define the regions in $\vec{m}$-space

also define

$$
\begin{aligned}
& A=\left\{\vec{m}: m_{i} \geqq t\right\}, \\
& B=\left\{\vec{m}: \frac{1}{N} \sum_{i=1}^{N} m_{i}>\bar{m}\right\} \cap A, \\
& C\left\{\vec{m}: \frac{1}{N} \sum_{i=1}^{N} m_{i} \leqq \bar{m}\right\} \cap A ;
\end{aligned}
$$

$$
I_{N}\left[\mathscr{C}^{N}\right]=\int_{\mathscr{C}^{N}} \prod_{j=1}^{N} d \omega_{j}\left|\operatorname{det} \omega_{j}\right| \exp \left(-\frac{1}{2}(\vec{\omega}-\vec{G})^{T} Y(\vec{\omega}-\vec{G})\right),
$$

where $\mathscr{C}^{N}=\mathscr{C} \times \mathscr{C} \times \cdots \times \mathscr{C}$ (note that $I_{N}$ differs from $I_{N}^{\prime}$ by using $\left|\operatorname{det} \omega_{j}\right|$ rather than $(-1)^{D} \operatorname{det} \omega_{j}$; cf. Eq. (34)), and

$$
\begin{aligned}
& P_{N}[\mathscr{D} ; A]=k_{N} \int_{A} \prod_{j=1}^{N} d m_{j} \exp \left(-\frac{1}{2} \vec{m}^{T} \bar{A}^{-1} \vec{m}\right) I_{N}\left[\mathscr{D}^{N}\right], \\
& P_{N}^{\prime}[\mathscr{D} ; A]=k_{N} \int_{A} \prod_{j=1}^{N} d m_{j} \exp \left(-\frac{1}{2} \vec{m}^{T} \bar{A}^{-1} \vec{m}\right) I_{N}^{\prime}\left[\mathscr{D}^{N}\right],
\end{aligned}
$$

where $k_{N}=\left((2 \pi)^{N d} \operatorname{det} \Lambda\right)^{-1 / 2}$. Thus

$$
P_{N}\left(\vec{x}_{1}, \ldots, \vec{x}_{N}\right)=P_{N}[\mathscr{D} ; A]
$$

and

$$
P_{N}^{\prime}\left(\vec{x}_{1}, \ldots, \vec{x}_{N}\right)=P_{N}^{\prime}\left[\mathbb{R}^{(1 / 2) D(D+1)} ; A\right] \text {. }
$$

I. First we obtain an estimate for $\left|P_{N}[\mathscr{D} ; C]-P_{N}^{\prime}\left[\mathbb{R}^{(1 / 2) D(D+1)} ; C\right]\right|$. For $\omega_{i}^{a b} \in \mathscr{D}$, $\left|\operatorname{det} \omega_{i}\right|=(-)^{D} \operatorname{det} \omega_{i}$, so $P_{N}[\mathscr{D} ; C]=P_{N}^{\prime}[\mathscr{D} ; C]$. Hence we may estimate $\left|P_{N}^{\prime}[\mathscr{D} ; C]-P_{N}^{\prime}\left[\mathbb{R}^{(1 / 2) D(D+1)} ; C\right]\right| \leqq k_{N} \int_{C} \prod_{i} d m_{j} e^{-(1 / 2) \vec{m}^{T} \bar{A}^{-1} \dot{m}}\left|I_{N}^{\prime}\left[\mathbb{R}^{(1 / 2) N D(D+1)}-\mathscr{D}^{N}\right]\right|$.

However, $\left|I_{N}^{\prime}\right| \leqq I_{N}$ by the triangle inequality, so we focus on $I_{N}\left[\mathbb{R}^{(1 / 2) N D(D+1)}-\mathscr{D}^{N}\right]$, restricted to the region $\vec{m} \in C$. Claim: there exists a sphere in $\vec{\omega}$-space centred about $\vec{G}$

\footnotetext{
6 To be more precise, $Q_{N D}^{(1)}(\alpha t)$ is a polynomial in $\alpha t, r, \alpha a^{-1 / 2}$ and $y^{-1 / 2}$ of degree $N D$, where $a, y$ are the eigenvalues of $\bar{A}^{-1}, Y$ respectively. The coefficients in $Q_{N D}^{(1)}$ depend only "weakly" on $\bar{A}^{-1}, Y$, i.e. via the matrix elements of the orthogonal transformations which diagonalize them. Similar remarks apply to $Q_{N D}^{(2)}$
} 
with radius $R=\frac{1}{2}[\eta /(1+\eta)] \alpha t$ that lies wholly within $\mathscr{D}^{N}$. For $\vec{m} \in C$,

and

$$
G_{i}^{a a}=-\alpha m_{i}+\varepsilon_{i j}^{a a} m_{j} \leqq-\alpha t+\varepsilon N \bar{m}
$$

$$
\left|G_{i}^{a b}\right|=\left|\varepsilon_{i j}^{a b} m_{j}\right| \leqq \varepsilon N \bar{m} \quad \text { if } a \neq b .
$$

Furthermore, for $\vec{\omega}$ 's within the sphere

one has

$$
\|\vec{\omega}-\vec{G}\|^{2}=\sum_{\substack{l \leq N \\ a \leqq b \leqq D}}\left|\omega_{i}^{a b}-G_{i}^{a b}\right|^{2}<R^{2},
$$

and

$$
\omega_{i}^{a a}<G_{i}^{a a}+R
$$

$$
\left|\omega_{i}^{a b}\right|<\left|G_{i}^{a b}\right|+R .
$$

Combining (83) with (86) gives

$$
-\omega_{i}^{a a}>-G_{i}^{a a}-R \geqq \alpha t-\varepsilon N \bar{m}-R,
$$

and combining (84) with (87) gives

$$
\left|\omega_{i}^{a b}\right|<\varepsilon N \bar{m}+R=\eta(\alpha t-\varepsilon N \bar{m}-R) .
$$

Then (88) and (89) imply

$$
\left|\omega_{i}^{a b}\right|<\eta \sqrt{\omega_{i}^{a a} \omega_{i}^{b b}},
$$

which guarantees via Lemma 1 that $\omega_{i} \in \mathscr{D}$, proving the claim.

Let $\mathscr{B}$ denote the set of $\vec{\omega}$ 's satisfying Eq. (85). Since $\mathscr{B} \subset \mathscr{D}^{N}$, the positivity of the integrand in $I_{N}$ implies

$$
0<I_{N}\left[\mathbb{R}^{(1 / 2) N D(D+1)}\right]-I_{N}\left[\mathscr{D}^{N}\right]<I_{N}\left[\mathbb{R}^{(1 / 2) N D(D+1)}-\mathscr{B}\right] .
$$

To estimate the latter, let $Y$ be diagonalized by the $S O\left(\frac{1}{2} N D(D+1)\right)$ transformation

$$
Y=O^{T} \bar{Y} O \text {. }
$$

After the change of variables $\vec{\omega}=O^{T} \vec{\omega}^{\prime}$,

$$
I_{N}\left[\mathbb{R}^{(1 / 2) N D(D+1)}-\mathscr{B}\right]=\int d \vec{\omega}^{\prime} \prod_{j=1}^{N}\left|\operatorname{det}\left(O^{T} \vec{\omega}^{\prime}\right)_{j}\right| \exp \left(-\frac{1}{2}\left(\vec{\omega}^{\prime}-\vec{G}^{\prime}\right)^{T} \bar{Y}\left(\vec{\omega}^{\prime}-\vec{G}^{\prime}\right)\right)
$$

where $\vec{G}^{\prime}=O \vec{G}$. Since $O$ is a rotation, the transformed integration region is $\mathbb{R}^{(1 / 2) N D(D+1)}-\mathscr{B}^{\prime}$, where $\mathscr{B}^{\prime}$ is the sphere of radius $R$ centred about $\vec{\omega}^{\prime}=\vec{G}^{\prime}$. Within $\mathscr{B}^{\prime}$ we can inscribe a hypercube of length $2 r, r=\left[\frac{1}{2} N D(D+1)\right]^{-1 / 2} R$. Then, expressing the product of determinants as a sum of monomials, we have the bound

$$
I_{N}\left[\mathbb{R}^{M}-\mathscr{B}\right]<\sum_{i_{1}, \ldots, i_{M}} C_{i_{1}, \ldots, l_{M}}\left[\prod_{\alpha=1}^{M}\left(\int_{-\infty}^{G_{\alpha}^{\prime}-r}+\int_{G_{\alpha}^{\prime}+r}^{\infty}\right) d \omega_{\alpha}^{\prime}\left|\omega_{\alpha}^{\prime}\right|^{\prime \prime} \exp \left(-\frac{1}{2}\left(\omega_{\alpha}^{\prime}-G_{\alpha}^{\prime}\right)^{2} \bar{Y}_{\alpha \alpha}\right)\right],
$$

where $M=\frac{1}{2} N D(D+1)$ and we have labeled the components of $\vec{\omega}^{\prime}$ and $\vec{G}^{\prime}$ by $\omega_{\alpha}^{\prime}$ and $G_{\alpha}^{\prime}, \alpha=1, \ldots, M$. Since each determinant has $D$ factors of $\omega_{\alpha}^{\prime}$, there are ND factors of $\omega_{\alpha}^{\prime}$ in each term of Eq. (94) so that $C_{i_{1}, \ldots, l_{M}}=0$ unless $\sum_{\alpha=1}^{M} i_{\alpha}=N D$, in which case 
$C_{i_{1}, \ldots, i_{M}}$ is just a number of order unity. Lemma 2 can be applied for each integral in Eq. (94), with the result

$$
I_{N}\left[\mathbb{R}^{M}-\mathscr{B}\right]<\frac{\exp \left(-\frac{1}{2} r^{2} \operatorname{tr} Y\right)}{r^{M} \operatorname{det} Y} Q_{N D}\left(r,\left|G_{\alpha}^{\prime}\right|, \bar{Y}_{\alpha \alpha}^{-1 / 2}\right)
$$

with $Q_{N D}$ being a polynomial of total degree $N D$ in its arguments.

Combining the results (95), (91) and (82) gives

$$
\begin{gathered}
\left|P_{N}[\mathscr{D} ; C]-P_{N}^{\prime}\left[\mathbb{R}^{(1 / 2) D(D+1)} ; C\right]\right|<\frac{k_{N} \exp \left(-\frac{1}{2} r^{2} \operatorname{tr} Y\right)}{r^{M} \operatorname{det} Y} \\
\cdot \int_{C} \prod_{j} d m_{j} e^{-(1 / 2) \vec{m}^{T} \bar{A}^{-1} \vec{m}} Q_{N D}\left(r,\left|G_{\alpha}^{\prime}\right|, \bar{Y}_{\alpha \alpha}^{-1 / 2}\right) .
\end{gathered}
$$

To estimate the integral in Eq. (96) the same technique may be applied as that used to bound Eq. (93). In this case the region $C$ is a distance $R=\sqrt{N} t$ from the point $\vec{m}=0$ where the exponential factor is maximized. Note that $G_{\alpha}^{\prime}$ is linear in the $m_{i}$ (Eq. (39a)). After applying Lemma 2 again we have

$$
\begin{gathered}
\left|P_{N}[\mathscr{D} ; C]-P_{N}^{\prime}\left[\mathbb{R}^{(1 / 2) D(D+1)} ; C\right]\right|< \\
k_{N} \frac{\exp \left(-\frac{1}{2} r^{2} \operatorname{tr} Y\right)}{r^{M} \operatorname{det} Y} \frac{\exp \left(-\frac{1}{2} t^{2} \operatorname{tr} \bar{A}^{-1}\right)}{t^{N} \operatorname{det} \bar{A}^{-1}} \\
\cdot Q_{N D}^{(1)}\left(\alpha t, r, \bar{Y}_{\alpha \alpha}^{-1 / 2}, \alpha a_{j}^{-1 / 2}\right),
\end{gathered}
$$

where $Q_{N D}^{(1)}$ is of degree $N D$ and $a_{j}$ are the eigenvalues of $\bar{A}^{-1}$. (We have suppressed the weak non-polynomial dependence on $Y$ and $\bar{A}^{-1}$ that comes from the orthogonal matrices which diagonalize them, as well as the dependence on $\varepsilon_{k l}^{i j}$, which can always be bounded by replacing $\varepsilon$ with $\alpha$ as we have done.) Since $r \propto \alpha t$ these two arguments may be lumped together in $Q_{N D}^{(1)}$.

II. The next step is to estimate $\left|P_{N}[\mathscr{D} ; B]-P_{N}^{\prime}\left[\mathbb{R}^{(1 / 2) D(D+1)} ; B\right]\right|$, or equivalently,

$$
\begin{aligned}
& \left|P_{N}^{\prime}[\mathscr{D} ; B]-P_{N}^{\prime}\left[\mathbb{R}^{(1 / 2) D(D+1)} ; B\right]\right| \\
& \quad \leqq k_{N} \int_{B} \prod_{i} d m_{j} \exp \left(-\frac{1}{2} \vec{m}^{T} \bar{A}^{-1} \vec{m}\right)\left|I_{N}^{\prime}\left[\mathbb{R}^{(1 / 2) N D(D+1)}-\mathscr{D}^{N}\right]\right| .
\end{aligned}
$$

For $\vec{m} \in B$, only weak bounds are available for $I_{N}^{\prime}$, since $G_{i}^{a b}$ is not necessarily negative definite. So we use

$$
\left|I_{N}^{\prime}\left[\mathbb{R}^{(1 / 2) N D(D+1)}-\mathscr{D}^{N}\right]\right| \leqq I_{N}\left[\mathbb{R}^{(1 / 2) N D(D+1)}-\mathscr{D}^{N}\right]<I_{N}\left[\mathbb{R}^{(1 / 2) N D(D+1)}\right]
$$

and, similarly to Eq. (94),

$$
\begin{aligned}
I_{N}\left[\mathbb{R}^{M}\right] & =\sum_{i_{1}, \ldots, i_{M}} C_{i_{1}, \ldots, i_{M}} \prod_{\alpha=1}^{M} \int_{-\infty}^{\infty} d \omega_{\alpha}^{\prime}\left|\omega_{\alpha}^{\prime}+G_{\alpha}^{\prime}\right|^{l_{\alpha}} \exp \left(-\frac{1}{2}\left(\omega_{\alpha}^{\prime}\right)^{2} \bar{Y}_{\alpha \alpha}\right) \\
& =\frac{Q_{N D}\left(G_{\alpha}^{\prime}, \bar{Y}_{\alpha \alpha}^{-1 / 2}\right)}{(\operatorname{det} Y)^{1 / 2}}
\end{aligned}
$$

by standard Gaussian integration, with $Q_{N D}$, once again, a polynomial of degree $N D$. Equation (100) is substituted into (98) via (99) and the $\vec{m}$ integral is bounded by the same technique as in part I of the proof. In particular, the region $B$ lies a distance 
$R=\sqrt{N} \bar{m}$ away from the point $\vec{m}=0$. Therefore, repeating the steps of part I leads to

$$
\begin{aligned}
& \left|P_{N}[\mathscr{D} ; B]-P_{N}^{\prime}\left[\mathbb{R}^{(1 / 2) D(D+1)} ; B\right]\right| \\
& \quad<\frac{k_{N}}{(\operatorname{det} Y)^{1 / 2}} \frac{\exp \left(-\frac{1}{2} \bar{m}^{2} \operatorname{tr} \bar{A}^{-1}\right)}{\bar{m}^{N} \operatorname{det} \bar{A}^{-1}} Q_{N D}^{(2)}\left(\alpha \bar{m}, \bar{Y}_{\alpha \alpha}^{-1 / 2}, \alpha a^{-1 / 2}\right) .
\end{aligned}
$$

III. The triangle inequality now implies

$$
\begin{aligned}
& \left|P_{N}[\mathscr{D} ; A]-P_{N}^{\prime}\left[\mathbb{R}^{(1 / 2) D(D+1)} ; A\right]\right| \\
& \quad=\left|P_{N}[\mathscr{D} ; B]+P_{N}[\mathscr{D} ; C]-P_{N}^{\prime}\left[\mathbb{R}^{(1 / 2) D(D+1)} ; B\right]-P_{N}^{\prime}\left[\mathbb{R}^{(1 / 2) D(D+1)} ; C\right]\right| \\
& \quad \leqq\left|P_{N}[\mathscr{D} ; B]-P_{N}^{\prime}\left[\mathbb{R}^{(1 / 2) D(D+1)} ; B\right]\right|+\left|P_{N}[\mathscr{D} ; C]-P_{N}^{\prime}\left[\mathbb{R}^{(1 / 2) D(D+1)} ; C\right]\right| .
\end{aligned}
$$

Using Eqs. (81), (97), and (101) gives us

$$
\begin{aligned}
& \left|P_{N}\left(\vec{x}_{1}, \ldots, \vec{x}_{N}\right)-P_{N}^{\prime}\left(\vec{x}_{1}, \ldots, \vec{x}_{N}\right)\right|<\frac{k_{N}}{(\operatorname{det} Y)^{1 / 2}} t^{-N}\left(\operatorname{det} \bar{A}^{-1}\right)^{-1} \\
& \quad \cdot\left\{\frac{\exp \left(-\frac{1}{2} r^{2} \operatorname{tr} Y-\frac{1}{2} t^{2} \operatorname{tr} \bar{A}^{-1}\right)}{r^{M}(\operatorname{det} Y)^{1 / 2}} Q_{N D}^{(1)}(\alpha t)+\frac{\exp \left(-\frac{1}{2} \bar{m}^{2} \operatorname{tr} \bar{A}^{-1}\right)}{(\bar{m} / t)^{N}} Q_{N D}^{(2)}(\alpha \bar{m})\right\} .
\end{aligned}
$$

The theorem then follows, using the definition of $k_{N}$ (below Eq. (80)) and Eqs. (50), (74).

By extracting the leading $t$-dependence of $P_{N}^{\prime}$, Eq. (53), and $\left|P_{N}-P_{N}^{\prime}\right|$, Eq. (72), the fractional error may be expressed as

$$
\begin{aligned}
\frac{\left|P_{N}-P_{N}^{\prime}\right|}{P_{N}^{\prime}} \sim & \exp \left(\frac{1}{2} t^{2} \sum_{i \neq j} \bar{A}_{i j}^{-1}\right)\left\{\frac{\exp \left(-\frac{1}{2} r^{2} \operatorname{tr} Y\right)}{r^{(1 / 2) N D(D+1)}(\operatorname{det} Y)^{1 / 2}}\right. \\
& \left.+\left(\frac{\alpha}{2 N \varepsilon}\left(\frac{\eta}{1+\eta}\right)\right)^{N(D-1)} \exp \left(-\frac{1}{2}\left(\bar{m}^{2}-t^{2}\right) \operatorname{tr} \bar{A}^{-1}\right)\right\} .
\end{aligned}
$$

It should be kept in mind that for large separations, $\left|\vec{x}_{i}-\vec{x}_{j}\right| \rightarrow \infty, \bar{A}_{i j}^{-1^{\prime}} \rightarrow 0$ for $i \neq j$ and $\bar{m} \rightarrow \infty$, since $\varepsilon \rightarrow 0$; however $\operatorname{tr} \bar{A}^{-1}$ and $\operatorname{tr} Y$ remain finite-see below. Therefore the relative error can be made arbitrarily small by going to sufficiently large distances and thresholds.

For the density of peaks above threshold, $N=1$, the relative error is more strongly bounded, for in this case $\bar{A}_{i j}^{-1}$ is by definition zero unless $i=j=1$, and similarly $\varepsilon_{i j}^{a b} \equiv 0$, so $\bar{m}=\infty$ and the second term in (104) vanishes. Thus the density of saddle points and local minima above threshold is exponentially suppressed relative to peaks, in accord with intuition.

To complete this section, we demonstrate the positivity of $\bar{A}^{-1}$ and $Y$, at least in the regime of large separations. As $\left|\vec{x}_{i}-\vec{x}_{j}\right| \rightarrow \infty$,

recall Eq. (58). Similarly,

$$
\bar{A}_{i j}^{-1} \rightarrow \delta_{i j} / \xi(0) ;
$$

$$
Y_{i j}^{a b, c d} \rightarrow \delta_{i j} Y_{(1)}^{a b, c d},
$$




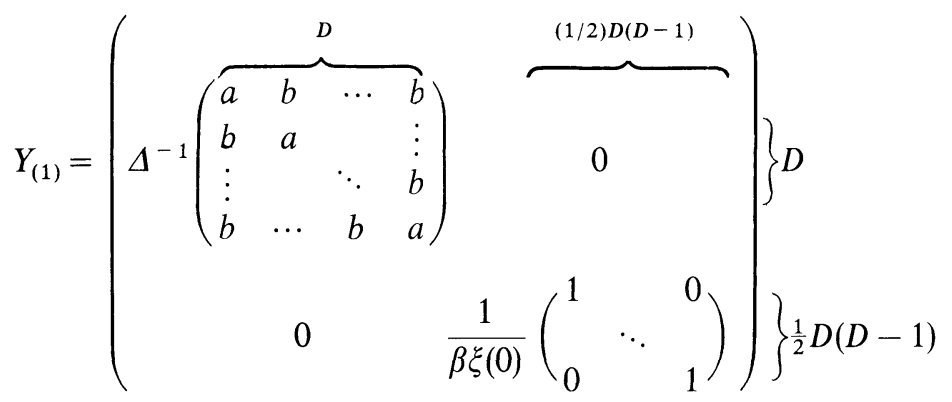

where $\beta=\nabla^{4} \xi(0) /[D(D+2) \xi(0)], a=(2 \beta)^{D-2}\left[(D+1) \beta-(D-1) \alpha^{2}\right], b=(2 \beta)^{D-2} \times$ $\left(\alpha^{2}-\beta\right), \Delta=(2 \beta)^{D-1} \xi(0)\left[(D+2) \beta-D \alpha^{2}\right]$. This can be diagonalized to obtain

$$
\bar{Y}_{(1)}=\frac{1}{\beta \xi(0)} \operatorname{diag} \underbrace{\left(\frac{1}{2} D^{2}(1-S)^{-1}\right.}_{1}, \underbrace{\frac{1}{2}, \ldots, \frac{1}{2}}_{D-1}, \underbrace{1, \ldots, 1)}_{1 / 2 D(D-1)},
$$

where

$$
S=\frac{D}{D+2} \frac{\alpha^{2}}{\beta}=\frac{\left(\nabla^{2} \xi(0)\right)^{2}}{\xi(0) \nabla^{4} \xi(0)}
$$

In order for the first eigenvalue of $\bar{Y}_{(1)}$ to be positive we require that $S \leqq 1$. In Fourier space this condition can be expressed as

where

$$
\left\langle k^{2}, 1\right\rangle^{2} \leqq\left\langle k^{2}, k^{2}\right\rangle\langle 1,1\rangle
$$

$$
\langle A, B\rangle=\left(\int d^{D} k A B \tilde{\xi}(k)\right) /\left(\int d^{D} k \tilde{\xi}(k)\right) .
$$

As long as $\tilde{\xi}(k)>0,\langle A, B\rangle$ is a positive definite inner product, and Schwarz's inequality implies that (110) is true, hence $S \leqq 1$. However, the probability measure in Eq. (2) may be written as

$$
P[\varepsilon]=\frac{1}{Z} \exp \left[-\frac{1}{2} \int \frac{d^{D} k}{(2 \pi)^{D}}|\varepsilon(k)|^{2} / \tilde{\xi}(k)\right] .
$$

In order for this to be normalizable (i.e., the Gaussian integral converges) it must be that $\tilde{\xi}(k)>0$. Thus $\bar{Y}$ is positive definite at infinite separations, as well as $\bar{A}^{-1}$. This conclusion must still be true at large finite separations since the eigenvalues only change by a factor of $1+O\left(\hat{\xi}_{i j}^{2}\right)$ (note Eq. (58) where the diagonal components are unaffected at $\left.O\left(\hat{\xi}_{i j}\right)\right)$.

\section{Appendix}

The matrix $\Lambda$ of Eq. (23a) is decomposed into the block form Eq. (25). The submatrices $A, B, C$ are given by

$$
\begin{aligned}
A_{i j}^{a b}= & \left\{\delta_{a 1} \delta_{b 1}+\delta_{a 1}\left(1-\delta_{b 1}\right) \frac{\partial}{\partial x_{j}^{b-1}}\right. \\
& \left.+\delta_{b 1}\left(1-\delta_{a 1}\right) \frac{\partial}{\partial x_{i}^{a-1}}+\left(1-\delta_{a 1}\right)\left(1-\delta_{b 1}\right) \frac{\partial^{2}}{\partial x_{i}^{a-1} \partial x_{j}^{b-1}}\right\} \xi\left(\left|\vec{x}_{i}-\vec{x}_{j}\right|\right),
\end{aligned}
$$




$$
\begin{aligned}
B_{i, j}^{a b, c d} & =\frac{\partial^{4}}{\partial x_{i}^{a} \partial x_{i}^{b} \partial x_{j}^{c} \partial x_{j}^{d}} \xi\left(\left|\vec{x}_{i}-\vec{x}_{j}\right|\right), \\
C_{i, j}^{a, b c} & =\left\{\delta_{a 1} \frac{\partial^{2}}{\partial x_{j}^{b} \partial x_{j}^{c}}+\left(1-\delta_{a 1}\right) \frac{\partial^{3}}{\partial x_{i}^{a-1} \partial x_{j}^{b} \partial x_{j}^{c}}\right\} \xi\left(\left|\vec{x}_{i}-\vec{x}_{j}\right|\right), \\
\left(C^{T}\right)_{i, j}^{b c, a}= & C_{j, i}^{a, b c},
\end{aligned}
$$

where $x_{i}^{a}$ is the $a$ th component of $\vec{x}_{i}, a=1, \ldots, D$. In (A-2) the pair $a b$ takes values $a$ $\leqq b=1, \ldots, D$, reflecting the fact that $\omega_{i}^{a b}$ is a symmetric matrix (Eq. 19), and likewise for $c d$, and $b c$ in (A-3), (A-4). The indices $i$ and $j$ label the spatial positions $\vec{x}_{i}$ and $\vec{x}_{j}$ and so run from 1 to $N$ for the $N$-point correlations of peaks.

\section{References}

1. Rice, S. O.: Mathematical analysis of random noise. In: Selected papers on noise and stochastic processes. New York: Dover 1954

2. Kaiser, N.: Ap. J. 284, L9 (1984)

3. Bardeen, J. M., Bond, J. R., Kaiser, N., Szalay, A. S.: Ap. J. 304, 15 (1986)

4. Politzer, H. D., Wise, M. B.: Ap. J. 285, L1 (1984)

5. Jensen, L. G., Szalay, A. S.: Ap. J. 305, L5 (1986)

6. Berezin, F. A.: The method of second quantization. New York, London: Academic Press 1966

7. Adler, R. J.: The geometry of random fields. New York: Wiley 1981

8. Doroshkevich, A. G.: Astrophysics 6, 320 (1970)

9. Otto, S., Politzer, H. D., Wise, M. B.: Phys. Rev. Lett. 56, 1978 (1986)

10. Mirsky, L.: An introduction to linear algebra. Oxford: Oxford University Press 1963

Communicated by G. Parisi

Received January 12, 1987 
